\title{
Anderson Orthogonality Catastrophe in Disordered Systems
}

\author{
Yuval Gefen ${ }^{1}$, Richard Berkovits ${ }^{2}$, Igor V. Lerner ${ }^{3}$, Boris L. Altshuler ${ }^{4,5}$ \\ 1 Department of Condensed Matter Physics, The Weizmann Institute of Science, Rehovot 76100, Israel \\ ${ }^{2}$ The Minerva Center, Department of Physics, Bar-Ilan University, Ramat-Gan 52900, Israel \\ ${ }^{3}$ School of Physics and Astronomy, University of Birmingham, Birmingham B15 2TT, United Kingdom \\ ${ }^{4}$ NEC Research Institute, 4 Independence Way, Princeton, New Jersey 08540 \\ ${ }^{5}$ Department of Physics, Princeton University, Princeton, New Jersey 08545
}

(October 27, 2018)

\begin{abstract}
We study the Anderson orthogonality catastrophe (AOC) in finite conductors with diffusive disorder. The disorder averaged logarithm of $\chi$, the overlap between the ground states before and after adding a static impurity, is found to depend nonmonotonically on the disorder. In two dimensions $\left\langle\ln \chi^{-1}\right\rangle \propto \ln ^{2} N$ in the weak disorder limit, thus showing a stronger dependence on the number of electrons $N$ than in the canonical AOC. A very broad tail of the distribution of $\chi$, found numerically, is a signature of the importance of a few-level statistics at the Fermi energy.
\end{abstract}

The Anderson orthogonality catastrophe is ubiquitous in physics, underlying such disparate phenomena as the zero-bias anomaly in disordered systems, the fractional Quantum Hall effect and the metal-insulator transition [1]. The seminal paper of Anderson [2] addressed a system of non-interacting electrons, confined to a finite volume. No randomness (or complex geometry) were considered. The introduction of a static impurity modifies the many-particle ground state (GS) of the original system, such that the overlap between the original and the modified GS, $\tilde{\chi}=\left|\left\langle\Psi \mid \Psi^{\prime}\right\rangle\right|^{2}$, is bounded (from above) by

$$
\chi=\exp \left[-\frac{1}{2} \sum_{\varepsilon_{n} \leq 0, \varepsilon_{m^{\prime}}>0}\left|\left\langle n \mid m^{\prime}\right\rangle\right|^{2}\right] \equiv \exp [-\mathcal{I}] .
$$

Here $|n\rangle$ and $\left|m^{\prime}\right\rangle$ are the single-particle eigenstates of the original and the modified system respectively, with energies $\varepsilon_{n}, \varepsilon_{m^{\prime}}$ counted from the Fermi level; $\mathcal{I}$ is referred to as the Anderson integral. For a short range impurity whose potential has the strength $\lambda$ it was found [2] that

$$
\mathcal{I}=2 \pi^{2} \lambda^{2} \ln N
$$

where $\mathrm{N}$ is the number of electrons: hence a "catastrophe" as $N$ approaches the thermodynamic limit.

Since the AOC appears to be a rather general phenomenon, it is only natural to study it under more general circumstances. The importance of the inclusion of disorder is self-evident $[3$ [ 5 . Not only may the disorderaveraged value of the overlap be different from its value in a pure system, but it can also induce mesoscopic fluctuations of $\mathcal{I}$ which are not necessarily narrowly distributed. Furthermore, one may consider longer range impurities as well: in 2DEG systems impurities are located off the conducting layer, implying softer impurity potentials.

In this work we consider electronic systems in the presence of quenched impurities that give rise to diffusive motion of the electrons, with an additional impurity further introduced into the system. We address two main issues concerning the AOC caused by the additional impurity.

(i) We consider the average value of the Anderson integral as a function of $N$, of disorder, characterised by the electron transport mean free path, $\ell$, and of the range, $b$, of the added impurity parameterized by

$$
U_{0} \equiv \frac{2 \pi \lambda}{\nu} u(\mathbf{r})=\frac{2 \pi \lambda}{\nu}\left(\pi b^{2}\right)^{-d / 2} \mathrm{e}^{-r^{2} / b^{2}},
$$

where $\nu$ is the single-particle density of states. We find that in the weak disorder regime and for $p_{F}^{-1} \lesssim b \ll L$ in $2 \mathrm{~d}$ ( $L$ is the linear system size) and $\ell \lesssim b \ll L$ in $3 \mathrm{~d}$ [7]

$$
\langle\mathcal{I}\rangle=\left(\pi^{2} \lambda^{2} / g_{0}\right) \begin{cases}\ln ^{2} N, & d=2 \\ (\ell / b) \ln N, & d=3 .\end{cases}
$$

Here $g_{0}=2 \pi^{2} \nu D \equiv 2 \pi^{2} \nu v_{F} \ell / d$ is the conductance of a $d$-dimensional cube of size $\ell^{d}$. Thus, in $2 \mathrm{~d}$ the decrease with $N$ of the overlap $\chi$, Eq. (11), is faster than a power law, showing a stronger $N$ dependence than in the original AOC for clean systems. This is due to the diffusive character of electron motion which increases the probability of return to the added impurity and thus the sensitivity of the modified GS to it. The overlap decreases faster when disorder increases, while in the strong disorder regime (localization) it should practically disappear - hence a non-monotonic dependence of $\chi$ on $g_{0}$.

There exists also a ballistic contribution to the overlap,

$$
\langle\mathcal{I}\rangle=\left(2 \pi^{4} \lambda^{2} / g_{0}\right)(\ell / \pi b)^{d-1} \ln N .
$$

For $b \lesssim p_{F}^{-1}$ it goes over to the "clean" result (2). For $p_{F}^{-1}<b<\ell$ it dominates in $3 \mathrm{~d}$, while in $2 \mathrm{~d}$, for the maximal $N$ possible in the weak disorder regime $\left(\ln N \lesssim g_{0}\right.$, see below), the diffusive contribution (仼) prevails.

(ii) We observe the appearance of a wide distribution, $\rho(\chi)$, of the GS overlaps. Numerically we find it to be anomalously broad even on a logarithmic scale. We argue that the tails of the distribution are defined by the 
level statistics of the last occupied and the first unoccupied levels. By projecting the problem onto a two-level Hilbert space and using random matrix theory, we derive this distribution non-perturbatively and show it to be in good agreement with the numerical results. The underlying physics of this part is closely related to a recent study of the AOC in the context of parametrically varied random matrices [8].

The logarithm of the overlap integral, $\mathcal{I}$, can be exactly expressed in terms of Green's functions:

$$
\mathcal{I}=\frac{\lambda^{2}}{2 \nu^{2}} \int \mathrm{d} \mathbf{r} \mathrm{d} \mathbf{r}^{\prime}\left\{u(\mathbf{r}) u\left(\mathbf{r}^{\prime}\right) \int_{-\infty}^{0^{-}} \mathrm{d} \varepsilon \int_{0^{+}}^{\infty} \mathrm{d} \varepsilon^{\prime} \frac{g^{*}(\varepsilon) g\left(\varepsilon^{\prime}\right)}{\left(\varepsilon-\varepsilon^{\prime}-i 0\right)^{2}}\right\},
$$

where $g(\varepsilon) \equiv G^{R}\left(\varepsilon ; \mathbf{r}, \mathbf{r}^{\prime}\right)-G^{A}\left(\varepsilon ; \mathbf{r}, \mathbf{r}^{\prime}\right)$. Scattering from all the other impurities is implicitly included in the formally exact retarded and advanced Green's functions, $G^{R}$ and $G^{A}$. The presence of disorder does not have a significant impact on the mean value of the overlap $\mathcal{I}$ provided that the additional impurity is point-like i.e. $b \lesssim \lambda_{\mathrm{F}}$ in Eq. (3). Taking the limit $b \rightarrow 0$, i.e. $u(\mathbf{r})=\delta(\mathbf{r})$, one reduces the overlap logarithm (6) to

$$
\mathcal{I}=\frac{(2 \pi \lambda)^{2}}{2 \nu^{2}} \int_{-\infty}^{0^{-}} \mathrm{d} \varepsilon \int_{0^{+}}^{\infty} \mathrm{d} \varepsilon^{\prime} \frac{\nu(\varepsilon) \nu\left(\varepsilon^{\prime}\right)}{\left(\varepsilon-\varepsilon^{\prime}-i 0\right)^{2}} .
$$

Then, in the leading order in $1 / g_{0}$, the average $\langle\nu \nu\rangle$ is given by the disconnected contribution, $\langle\nu\rangle\langle\nu\rangle$. Approximating $\langle\nu(\varepsilon)\rangle$ by $\nu$ for $|\varepsilon|<E / 2$ ( $E$ is the bandwidth) and vanishing outside the band, one finds the average of (7) equal to the Anderson expression, $\langle\mathcal{I}\rangle=$ $2 \pi^{2} \lambda^{2} \ln (E / \Delta)$, where $\Delta=1 / \nu L^{d}$ is the mean singleparticle level spacing.

When the range of $u(\mathbf{r})$ is finite, the mean value of $\mathcal{I}$ is more strongly affected by disorder. Now one needs to take into account both the disconnected (short-range), $\left\langle g^{*}(\varepsilon)\right\rangle\left\langle g\left(\varepsilon^{\prime}\right)\right\rangle$, and connected (long-range), $\left\langle g^{*}(\varepsilon) g\left(\varepsilon^{\prime}\right)\right\rangle_{\mathrm{c}}$, contributions. The former is given by [9] 10$]$

$$
\langle g(\varepsilon)\rangle\left\langle g\left(\varepsilon^{\prime}\right)\right\rangle=(2 \pi \nu)^{2} e^{-R / \ell} \begin{cases}J_{0}^{2}\left(k_{F} R\right), & d=2 \\ \frac{\sin ^{2}\left(k_{F} R\right)}{\left(k_{F} R\right)^{2}}, & d=3,\end{cases}
$$

where $R=|\mathbf{R}| \equiv\left|\mathbf{r}-\mathbf{r}^{\prime}\right|$. Substituting Eq. (8) into Eq. (6), and performing the spatial integration and subsequently the energy integration yields Eq. (5).

The long-range contribution is obtained from Eq. (6) by considering the impurity average over the advancedretarded parts of $g^{*} g$ yielding

$$
\langle\mathcal{I}\rangle=2 \pi \lambda^{2} \Re \mathrm{e} \int_{\Delta}^{1 / \tau} \frac{\mathrm{d} \omega}{\omega} \Delta \sum_{\mathbf{q}} \int \mathrm{d} \mathbf{r} \mathrm{d} \mathbf{r}^{\prime} \frac{u(\mathbf{r}) u\left(\mathbf{r}^{\prime}\right) \mathrm{e}^{i \mathbf{q} \cdot \mathbf{R}}}{D q^{2}-i \omega} .
$$

Substituting the expression for $u$, Eq. (3), performing the spatial integration, replacing the summation over q by the integration and finally performing the integral over $\omega$ we obtain Eq. (\$), where we have used $\ln (L / b)^{2}=\ln N-\ln \left[\left(k_{F} b\right)^{2} / 4 \pi\right] \approx \ln N$ for $b \ll L[11$. The incipient AOC becomes negligibly small when the impurity is delocalized over the entire system.

We have thus found that once the impurity range exceeds $p_{F}^{-1}$ in $d=2$ or $\ell \gg p_{F}^{-1}$ in $d=3$, the main contribution to the overlap integral is the long-range term (4). In $d=2$, it gives rise to the suppression of $\chi$ which is stronger than a power law in $N$. Furthermore, the condition that the magnitude of this effect exceeds the original $\mathrm{AOC}$ factor (2) is

$$
L>\ell \exp g_{0}
$$

At the same time we require weak localization (WL) corrections (neglected here) to be small, i.e. $L<\xi$, where $\xi$ is the localization length. In the presence of time reversal (GOE) symmetry $\xi_{o} \sim \ell \exp g_{0}$ [12], and these two inequalities are only marginally compatible. On the other hand, for a broken (e.g., due to an applied magnetic field) time reversal (GUE) symmetry $\xi_{u} \sim \ell \exp g_{0}^{2}$, i.e. there exists a regime for which both Eq. (10) and the requirement on the smallness of WL corrections are satisfied.

Arguably the most intriguing result concerning $\langle\mathcal{I}\rangle$ is the dependence on disorder. It is clear from Eq. (化) that in the weak disorder limit (and finite impurity range) the exponential suppression of the overlap is enhanced as $g$ decreases. On the other hand one expects the AOC to vanish in the strongly localized limit. This implies a nonmonotonic dependence on disorder. Such a behavior is, indeed, found numerically (see Fig. 1).

The analysis outlined above concerns with the average value of $\mathcal{I}$. As we show below, the distribution function $\rho(\chi)$ is remarkably broadly shaped, so that the average alone does not provide us with the full information on the behavior of $\chi$.

Our numerical study addressed a tight-binding Hamiltonian in the presence of diagonal disorder: the site energies assumed a box distribution of width $W$. The nearestneighbor hopping, $t$, was chosen to be constant in magnitude, but with a phase factor when a magnetic field is applied. The underlying lattice was a two-dimensional square of area $L^{2}$ with periodic boundary conditions. For each realization of onsite energies the Hamiltonian is diagonalized exactly, both in the absence and in the presence of an additional impurity, parametrized according to Eq. (3). The calculation is performed for up to $5 \times 10^{4}, 3.2 \times 10^{4}, 2 \times 10^{4}, 1.6 \times 10^{4}, 1.2 \times 10^{4}$ different realizations for the sample sizes $L=16,20,24,28,32$, respectively, and several values of disorder.

The broad distribution of $\mathcal{I}$ (normalized by its average value $\langle\mathcal{I}\rangle$ ) is presented in Fig. 2 for different values of $L$ with filling $N / L^{2}=1 / 4$ and for $W=3 t$ and $W=2 t$. Here we consider an impurity with $\lambda=1$ and $b=2$. 
The distribution we find is quite remarkable. Clearly it is even broader than log-normal [13]. The extended tail of the distribution corresponds to small values of $\chi$ (large values of $\mathcal{I} /\langle\mathcal{I}\rangle$ ) and gives the appearance of power law behavior. The range of the tail shown in Fig. 2 (before the statistical scatter of data points washes it out) is at least 15 standard deviations. Furthermore the distribution seems to be governed by a single scaling parameter $\mathcal{I} /\langle\mathcal{I}\rangle$, and does not depend on system size or on disorder. The distributions exhibit extended tails and obey the single parameter scaling both for the GOE and the GUE symmetries. Upon close inspection it appears that the details of the tail differ between the GOE and the GUE scenarios, the occurrence of atypical events been suppressed in the latter (a "smaller tail"). This is accounted for below.

The clear deviation of the distribution's tail from a lognormal shape suggests that large fluctuations in $\mathcal{I}$ are a manifestation of few-level physics. This is supported by a direct diagrammatic estimation of fluctuations. The leading contributions to $\left\langle\mathcal{I}^{2}\right\rangle$ and to the higher cumulants of $\mathcal{I}$ come from the same diagrams as those for the local density of states (LdoS), as is clear from Eq. (6). The LDoS distribution by itself is log-normal [14]; the additional energy denominator in Eq. (6) leads to powers of (still) logarithmic divergence in all orders of perturbation theory for the cumulants of $\mathcal{I}$ higher than those for the LdoS cumulants. This indicates that the distribution of $\mathcal{I}$ is even wider than log-normal. Although it makes a direct diagrammatic (or renormalization group) analysis of the fluctuations of $\mathcal{I}$ hardly possible, this infrared divergence indirectly indicates the importance of just a few energy levels near the Fermi energy.

The final confirmation of the few level picture is provided by a scatter plot of $\chi$ vs. the levels spacing $\delta$ between the $N+1$ st and $N$ th level for the quarter filled case, Fig. 3. In these plots the differences (GOE vs. GUE) hinted to in the distribution plots become much clearer. While there is a finite (small) probability of finding small Anderson overlaps $(\chi<1 / 2)$ for the GOE case, there is almost no probability of finding such small overlaps in the GUE case. At the same time small values of $\chi$ are strongly correlated with small level spacings, leading to suppression of the tail of $\rho$ in the GUE case.

Since the level spacing at the Fermi energy is strongly correlated with small values of the Anderson integral, it is reasonable to try to develop an analytic description for the tail of the distribution from the behavior of a two-level system representing the last occupied and first unoccupied levels in the system [8]. Prior to the introduction of the impurity the levels have the energy $-\delta / 2$ and $\delta / 2$ and wave functions $\psi_{N}(\vec{r})$ and $\psi_{N+1}(\vec{r})$ respectively. After the introduction of an impurity $\lambda \delta(\vec{r}=0)$, the two level system is represented by the following Hamiltonian:

$$
H=\left(\begin{array}{cc}
\delta / 2+\lambda\left|\psi_{N+1}(0)\right|^{2} & \lambda \psi_{N+1}^{*}(0) \psi_{N}(0) \\
\lambda \psi_{N+1}(0) \psi_{N}^{*}(0) & -\delta / 2+\lambda\left|\psi_{N}(0)\right|^{2}
\end{array}\right) .
$$

One can bound $\chi$ from above by $\chi^{\prime}$, the contribution to the Anderson overlap due to the $N$-th level (before and after the introduction of the impurity). This is readily done by diagonalizinig the above Hamiltonian:

$$
\chi^{\prime}=\frac{1}{2}+\frac{1}{2} \frac{\delta+\lambda\left(\left|\psi_{N+1}(0)\right|^{2}-\left|\psi_{N}(0)\right|^{2}\right)}{\sqrt{\left(\delta / 2+\lambda\left(\left|\psi_{N+1}(0)\right|^{2}-\left|\psi_{N}(0)\right|^{2}\right) / 2\right)^{2}+\lambda^{2}\left|\psi_{N+1}(0)\right|^{2}\left|\psi_{N}(0)\right|^{2}}}
$$

We further assume that the distribution of the level spacing $\delta$ is given by

$P(\delta / \Delta)= \begin{cases}(\pi \delta / 2 \Delta) \exp \left[-(\pi / 4)(\delta / \Delta)^{2}\right], & \text { GOE } \\ \left(32 / \pi^{2}(\delta / \Delta)^{2}\right) \exp \left[-(4 / \pi)(\delta / \Delta)^{2}\right], & \text { GUE }\end{cases}$

and the wave function distributions are $P(\Re$ e $\psi(0))=$ $P(\Im \mathrm{m} \psi(0))=(\tilde{N} / 2 \pi)^{1 / 2} \exp \left(-\widetilde{N} \Re \mathrm{e} \psi(0)^{2} / 2\right)$ for the GUE case, while in the GOE case there are only real components of the wavefunction $\left(\widetilde{N} \sim\left(p_{F} L\right)^{2}\right)$. The distribution of $\chi^{\prime}$ is given then by

$$
\rho(\chi)=\int_{0}^{\infty} \mathrm{d} \delta P\left(\frac{\delta}{\Delta}\right) \int \mathrm{D} \psi_{N+1} \mathrm{D} \psi_{N} \delta\left(\chi^{\prime}-\chi\right)
$$

where $\chi^{\prime}$, which depends on all the integration variables, is given by Eq. (12), and $\mathrm{D} \psi_{N(N+1)}$ denotes an integration over the real and imaginary parts of $\psi_{N(N+1)}(0)$. The integration is performed numerically using a Monte Carlo method [15], and the results for the tail region are shown in the inset of Fig. 2. As can be seen, deep in the tail region $\rho(\chi)$ fits the distribution reasonably well.
To summarize, we have found three main peculiarities in the physics of AOC in diffusive $2 \mathrm{~d}$ systems. First, the average value of the overlap between the original and the modified ground states decreases with $N$ faster than any power law. Second, it shows a non-monotonic dependence on disorder. Finally, the logarithm of the overlap shows remarkably wide fluctuations with disorder, with long tails which are mainly due to the contributions from only a few levels near the Fermi energy.

We acknowledge discussions with C. Lewenkopf and R.O. Vallejos. This work was supported in part by the U.S.-Israel BSF, the GIF foundation, the ISF-Centers of Excellence Program, the Minerva foundation, and by the Leverhulme trust. Y.G., R.B. and I.V.L. acknowledge the kind hospitality extended to them at Princeton University and NEC. 
[1] The Altshuler-Aronov effect (JETP 50, 968 (1980)), the vanishing of the DOS at the metal-insulator transition (D. Vollhardt and P. Wölfle, Phys. Rev. Lett. 48, 699 (1982)) and the singularity of the tunneling DOS of FQHE systems (J.M. Kinaret, Y.Meir, N.S. Wingreen, P.A. Lee and X.G. Wen, Phys. Rev. B 46, 4681 (1992)) are all manifestations of the AOC.

[2] P. W. Anderson, Phys. Rev. Lett. 18, 1049 (1967).

[3] Y. Chen and J. Kroha, Phys. Rev. B 46, 1332 (1992).

[4] K. A. Matveev and I. L . Aleiner, Phys. Rev. Lett. 80, 814 (1998).

[5] I. E. Smolyarenko and B. D. Simons (unpublished).

[6] G. Montambaux and F. Piéchon (unpublished).

[7] There are corrections to the results (4) and (8) involving terms $\sim \ln k_{F} b$ and $\sim \ln g_{0}$ small compared to $\ln N$.

[8] R. O. Vallejos, C. Lewenkopf and Y. Gefen, to be published.

[9] A. D. Mirlin, Phys. Repts. B 326, 259 (2000).

[10] Note that since $\left\langle G^{R / A}\right\rangle$ is essentially energy independent we omit the dependence on $\varepsilon, \varepsilon^{\prime}$. More precisely, the $k_{F}$ factors in Eq. (8) should be replaced by $k(\varepsilon)$ or $k\left(\varepsilon^{\prime}\right)$ in the energy integrals. The $J_{0}^{2}$ or the $\sin ^{2}$ terms are then modified into oscillatory terms which effectively restrict the energy integrals to $\left|\varepsilon-\varepsilon^{\prime}\right| \leq \varepsilon_{F} / k_{F} b$. We end up with the $\ln N$ factors being replaced by $\ln \left(N / k_{F} b\right)[]$.

[11] Note that the long-range contribution arises from an energy shell $\sim D / b^{2} \leq \hbar / \tau$ around the Fermi energy in contrast to the original AOC or the short range term, which are the entire Fermi sea contributions.

[12] I. V. Lerner and Y. Imry, Europhys. Lett. 29, 49 (1995).

[13] By contrast the distribution of $1 / \mathcal{I}$, for which the tail probes values of $\chi$ close to one, is clearly log-normal and depends on the disorder.

[14] I. V. Lerner, Phys. Let. 133A, 253 (1988); A. D. Mirlin, Phys. Rev. B 53, 1186 (1996).

[15] One can simplify Eq. (13) to the level of a double integral which can then be computed numerically. A direct numerical evaluation of the integral Eq. (13) is faster.

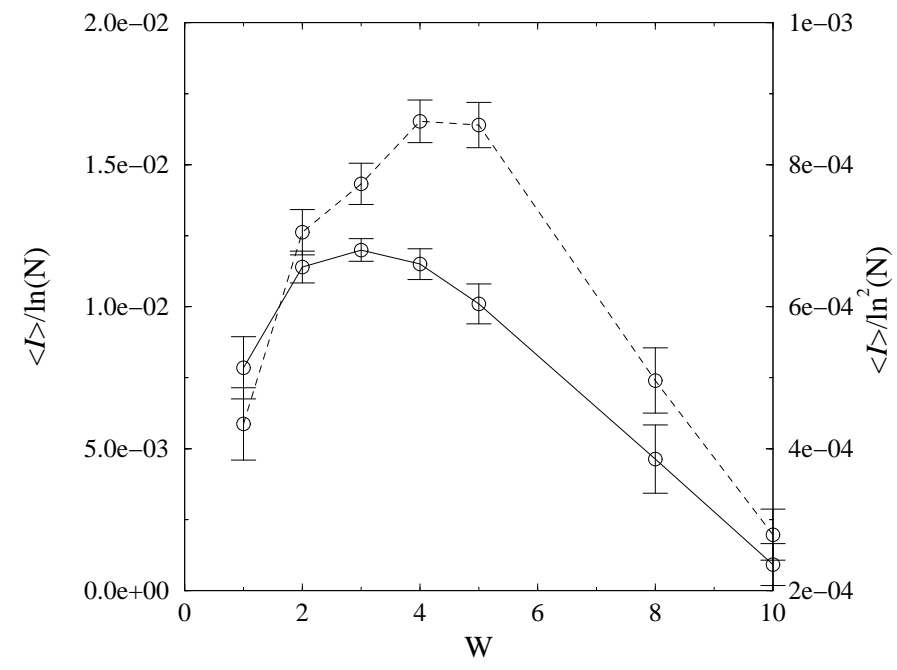

FIG. $\quad 1 .: \quad\left\langle\langle\mathcal{I}\rangle_{\text {disorder }} / \ln N\right\rangle_{N} \quad$ (solid line) and $\left\langle\langle\mathcal{I}\rangle_{\text {disorder }} / \ln ^{2} N\right\rangle_{N}$ (dashed line) vs. the disorder $W$ for an $L=32$ system around quarter filling.
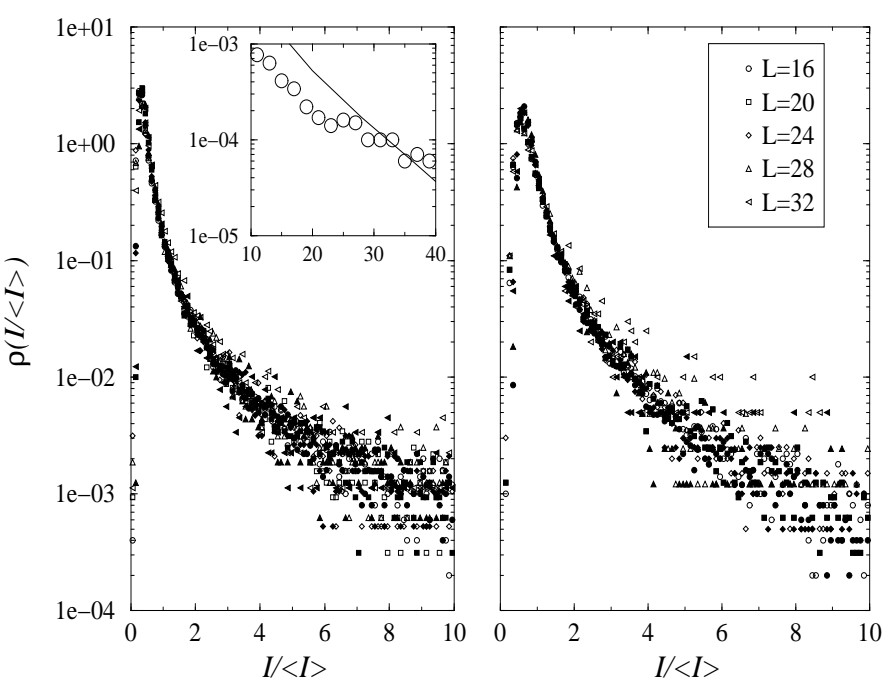

FIG. 2.: The distribution of $\mathcal{I}$ normalized by its average for different system sizes $L$ at quarter filling $N=L^{2} / 4$. Open symbols correspond to $W=2 t$ while filled ones to $W=3 t$. Left panel: No magnetic field (corresponding to GOE statistics). Right panel: Finite magnetic field (GUE statistics). Note that the larger values of $\mathcal{I} /\langle\mathcal{I}\rangle$ correspond to smaller values of $\chi$. Inset: The tail region for the GOE statistics at quarter filling, $L=16, W=3 t$. The line corresponds to the two-level theory.

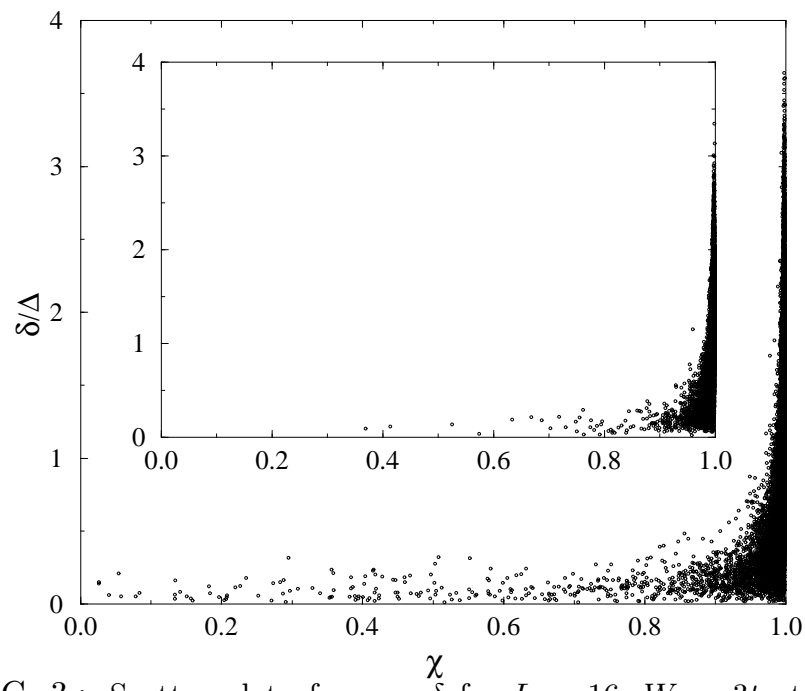

FIG. 3.: Scatter plot of $\chi$ vs. $\delta$ for $L=16, W=3 t$ at quarter filling with GOE statistics. Inset: GUE statistics. 